\title{
Video Article \\ Advanced Diffusion Imaging in The Hippocampus of Rats with Mild Traumatic Brain Injury
}

\author{
Kim Braeckman ${ }^{1}$, Benedicte Descamps ${ }^{1}$, Christian Vanhove ${ }^{1}$ \\ ${ }^{1}$ Infinity lab, Medical Imaging and Signal Processing Group, Ghent University
}

Correspondence to: Kim Braeckman at Kim.Braeckman@ugent.be

URL: https://www.jove.com/video/60012

DOI: doi:10.3791/60012

Keywords: Neuroscience, Issue 150, traumatic brain injury, agnetic resonance imaging, diffusion tensor imaging, preclinical, rat, hippocampus

Date Published: 8/14/2019

Citation: Braeckman, K., Descamps, B., Vanhove, C. Advanced Diffusion Imaging in The Hippocampus of Rats with Mild Traumatic Brain Injury. J. Vis. Exp. (150), e60012, doi:10.3791/60012 (2019).

\section{Abstract}

Mild traumatic brain injury ( $\mathrm{mTBI}$ ) is the most common type of acquired brain injury. Since patients with traumatic brain injury show a tremendous variability and heterogeneity (age, gender, type of trauma, other possible pathologies, etc.), animal models play a key role in unraveling factors that are limitations in clinical research. They provide a standardized and controlled setting to investigate the biological mechanisms of injury and repair following TBI. However, not all animal models mimic the diffuse and subtle nature of mTBI effectively. For example, the commonly used controlled cortical impact (CCl) and lateral fluid percussion injury (LFPI) models make use of a craniotomy to expose the brain and induce widespread focal trauma, which are not commonly seen in mTBI. Therefore, these experimental models are not valid to mimic mTBI. Thus, an appropriate model should be used to investigate mTBI. The Marmarou weight drop model for rats induces similar microstructural alterations and cognitive impairments as seen in patients who sustain mild trauma; therefore, this model was selected for this protocol. Conventional computed tomography and magnetic resonance imaging (MRI) scans commonly show no damage following a mild injury, because mTBI induces often only subtle and diffuse injuries. With diffusion weighted MRI, it is possible to investigate microstructural properties of brain tissue, which can provide more insight into the microscopic alterations following mild trauma. Therefore, the goal of this study is to obtain quantitative information of a selected region-of-interest (i.e., hippocampus) to follow up disease progression after obtaining a mild and diffuse brain injury.

\section{Video Link}

The video component of this article can be found at https://www.jove.com/video/60012/

\section{Introduction}

Traumatic brain injury (TBI) has gained more attention in recent years, as it has become clear that these brain injuries can result in lifelong cognitive, physical, emotional, and social consequences ${ }^{1}$. Despite this increasing awareness, mild TBI (mTBI, or concussion) is still often underreported and undiagnosed. MTBI has been referred to as a silent epidemic, and individuals with a history of mTBI show higher rates of substance abuse or psychiatric problems ${ }^{2}$. Several patients with $\mathrm{mTBI}$ go undiagnosed every year due to the diffuse and subtle nature of the injuries, which are often not visible on conventional computed tomography (CT) or magnetic resonance imaging (MRI) scans. This lack of radiological evidence of brain injury has led to the development of more advanced imaging techniques such as diffusion MRI, which are more sensitive to microstructural changes ${ }^{3}$.

Diffusion MRI allows in vivo mapping of the microstructure, and this MRI technique has been used extensively in TBI studies ${ }^{4,5,6}$. From the diffusion tensor, fractional anisotropy (FA) and mean diffusivity (MD) are computed to quantify alteration in the microstructural organization following injury. Recent reviews in mTBI patients report increases in FA and decreases in MD following injury, which can be indicative of axonal swelling ${ }^{7}$. Contrary, increases in MD and decreases in FA are also found and have been suggested to underlie disruptions in parenchymal structure following edema formation, axonal degeneration, or fiber misalignment/disruption ${ }^{8}$. These mixed findings can be partially explained by the significant clinical heterogeneity of $\mathrm{mTBI}$ caused by different types of impact and severity (e.g., rotation-acceleration, blunt force trauma, blast injury or combination of the former). However, currently there is no clear consensus about the underlying pathology and biological/cellular basis underpinning alterations in the microstructural organization.

Animal models provide a standardized and controlled setting to investigate biological mechanisms of injury and repair following TBI in greater detail. Several experimental models for TBI have been developed and represent different aspects of human TBI (e.g., focal vs. diffuse trauma or trauma caused by rotational forces $)^{9,10}$. Commonly used animal models include the controlled cortical impact (CCl) and lateral fluid percussion injury (LFPI) models ${ }^{11,12}$. Although the experimental parameters can be well-controlled, these models make use of a craniotomy to expose the brain. Craniotomies or skull fractures are not commonly seen in $\mathrm{mTBI}$; therefore, these experimental models are not valid to mimic mTBI. The impact acceleration model developed by Marmarou et al. ${ }^{13}$ makes use of a weight that is dropped from a certain height onto the rat's head, which is protected by a helmet. This animal model induces similar microstructural alterations and cognitive impairments as seen in patients who sustain mild trauma. Therefore, this Marmarou weight drop model is appropriate to investigate imaging biomarkers for diffuse mTBI ${ }^{14,15}$ 
This report demonstrates the application of advanced diffusion MRI in an $\mathrm{mTBI}$ rat model using the Marmarou weight drop model. First shown is how to induce a mild and diffuse trauma, and analysis using diffusion tensor imaging (DTI) model is then provided. Specific biological information is obtained with the use of more advanced diffusion models [i.e., diffusion kurtosis imaging (DKI) and white matter tract integrity (WMTI) model]. Specifically, mild trauma is inflicted and microstructural changes are then evaluated in the hippocampus using conventional T2-weighted MRI and an advanced diffusion imaging protocol.

\title{
Protocol
}

The protocol has been approved by the Animal Ethics Committee at the University of Ghent (ECD 15/44Aanv), and all experiments were conducted in accordance with the guidelines of the European Commission (Directive 2010/63/EU).

\section{Animal preparation and helmet attachment}

1. Weigh a female Wistar $\mathrm{H}$ rat $( \pm 250 \mathrm{~g}$ or 12 weeks of age) and anesthetize in a small induction chamber filled with a mixture of isoflurane $(5 \%)$ and $\mathrm{O}_{2}$ for at least $1 \mathrm{~min}$.

2. Inject the rat with $0.05 \mathrm{mg} / \mathrm{kg}$ buprenorphine subcutaneously in the neck, return it to the home cage, and allow pre-emptive analgesia for at least 30 min to take an effect.

NOTE: During the 30 min wait, the surgical site can be prepared.

3. Place a heating pad kept at $37^{\circ} \mathrm{C}$ under the surgical field. Place the sterilized surgical instruments on the surgical field that was disinfected with $70 \%$ ethanol.

4. Place the rat back in the induction chamber and anesthetize the rat until it is non-responsive to a paw or tail pinch.

5. Place the rat on the surgical field and insert a catheter in the lateral tail vein. Next, shave the rat's head, remove excess fur and disinfect the scalp and the rest of the surgical area with chlorohexidine.

6. Inject $100 \mu \mathrm{L}$ of $2 \%$ lidocaine locally in the scalp.

7. Make a midline incision using a scalpel size 11 to expose the skull, removing any excess membranes with small scissors. Retract the skin using an ocular speculum with a maximum spread of $1 \mathrm{~cm}$. Additionally, remove the periosteum by gently rubbing a sterile cotton bud across the skull until the periosteum is no longer present.

8. Put one drop of tissue glue on the skull and one on the sterilized metallic disc (diameter of $10 \mathrm{~mm}$ and $3 \mathrm{~mm}$ thickness), which acts as the helmet. Glue the disc approximately one-third before and two-thirds behind the bregma. Allow the glue to dry for $1 \mathrm{~min}$.

\section{Induction of traumatic brain injury (TBI)}

1. Place the rat on the custom-made bed with a foam mattress of certain spring constant (see Table of Materials). Position the rat directly under a transparent plastic tube with a $450 \mathrm{~g}$ brass weight with the helmet as horizontal as possible. Detach the rat from anesthesia.

2. Pull the weight up to $1 \mathrm{~m}$ and release when ready. Ensure that a second experimenter is present to move the rat away from the plastic tube immediately after impact to prevent a second impact. NOTE: The sham injured rats receive the same experimental procedure (steps 1.1-2.7), except for step 2.2.

3. Re-attach the rat to the anesthesia and inject $1 \mathrm{~mL}$ of physiological solution $(0.9 \% \mathrm{NaCl})$ via the catheter to reduce the hemodynamic shock NOTE: It is possible that the rat briefly stops breathing because of the impact. Gently compress the thorax if the rat does not spontaneously breathe after $2 \mathrm{~s}$ to encourage the breathing reflex.

4. Remove the helmet by gently pulling it from the skull. Remove any remaining glue from the skull and skin and close the incision with surgical suture. Apply local analgesia gel using a sterile applicator tip.

5. Place the rat on the bed of the CT scanner. Confirm the correct position using a scout scan. Adjust the field of view to enable imaging of the whole head within one bed position. Administer a general purpose, low dose CT scan to rule out skull fractures. NOTE: Skull fracture is a criterium for euthanasia.

6. Place the rat in a clean cage on a heating pad $\left(37^{\circ} \mathrm{C}\right)$. Monitor the time to regain consciousness. Once the rat is able to sit upright, the rat can be returned to the home cage.

7. Administer a second dose of $0.05 \mathrm{mg} / \mathrm{kg}$ buprenorphine one day following TBI induction.

\section{Diffusion magnetic resonance imaging (MRI)}

\author{
NOTE: Diffusion-weighted imaging is performed before and 1 day following trauma induction.
}

1. Anesthetize the rat in a small induction chamber filled with a mixture of isoflurane $(5 \%)$ and $\mathrm{O}_{2}$. When the rat is non-responsive to a paw or tail pinch reduce the anesthesia to $2 \%$ with a flow rate of $500 \mathrm{~mL} / \mathrm{min}$. Transfer the animal to the scanner bed in head-first prone position.

2. Position the rat in the head holder with the teeth bar and nose cone, delivering the anesthesia, and slide the head forwards until the center of the brain is at the level of the center of the quadrature volume MRI coil. Apply lubricating ointment to the eyes in small amounts to prevent any damage to the cornea. Fixate the head with a small piece of tape to avoid movement during scanning.

3. Place a pressure pad under the thorax of the rat to monitor respiration and cover the rat with a circulating warm water heating blanket and bubble wrap to keep the rat warm. Before the scanning, check the respiratory monitor to ensure that the signal is clear without noise and that the respiratory cycle is consistent. Relocate the pressure pad, if necessary. NOTE: The respiratory rate should be kept between 1 breath per 1,200-1,700 ms by adjusting the level of anesthesia between $1 \%-2 \%$.

4. Slide the quadrature volume coil over the head. Adjust the tuning and matching capacitors of the coil to the proper frequency and impedance according to the instructions provided by the coil vendor. Advance the scanner bed into the scanner bore to start scanning.

5. Obtain a default three-plane scout scan ("tripilot") to ensure correct positioning.

1. Load the tripilot sequence into the Scan Control by clicking New Scan and selecting the tripilot sequence from the protocol list. Next, click the traffic light button to start the scan. 
2. When the scan is finished, load the scan in the image display and ensure that 1) the head is lying straight and 2) the brain is positioned in the center of the magnet and coil. Adjust the position of the head and/or the scanner bed, if necessary, and acquire a new tripilot scan.

6. Adjust the local magnetic field using an automated second-order shimming protocol: load the second-order shim protocol into the Scan Control as described in step 3.5.1. Next, click on the Acq tab | Current Adjustments | Method specific adjustment for the Local Field Homogeneity in the Spectrometer Control Tool window to start automated shimming.

7. Load a new T2 Rapid imaging with Refocused Echoes (RARE) sequence into the Scan control as described in step 3.5.1.

1. Acquire T2 weighted images using the default settings, except for the following parameters:

2. Open the Edit Scan tab and adjust the repetition time (TR) and echo time (TE) to $5,500 \mathrm{~ms}$ and 37 ms, respectively. Also, modify the field of view and matrix size to allow for a higher in-plane resolution of $109 \mu \mathrm{m} \times 109 \mu \mathrm{m}$ (default resolution $=156 \mu \mathrm{m} \times 156 \mu \mathrm{m}$ ). Make sure that the slice thickness is $600 \mu \mathrm{m}$, number of slices is set to 45 , and RARE factor is set to 8 .

3. Open the Geometry editor and place the slice package in the correct position including the bulbus of the brain and the cerebellum.

8. Load three new echo-planar diffusion-weighted spin-echo sequence (DtiEpi) from the B_DIFFUSION folder into the Scan Control protocol as described in step 3.5.1.

NOTE: Using three different diffusion "shells", the diffusion tensor imaging (DTI) model ${ }^{4,16}$, diffusion kurtosis imaging (DKI) model ${ }^{17}$, and white matter tract integrity (WMTI) model ${ }^{18}$ can all be estimated. It is recommended to use at least three different b-values, with the highest $b$-value having a maximum of $3000 \mathrm{~s} / \mathrm{mm}^{2}$ with at least 15 evenly spaced directions per imaging shell ${ }^{17}$.

1. Acquire diffusion weighted images (DWls) using default settings, apart from the following settings:

2. Open the Edit Scan tab and adjust the geometrical parameters under the Geometry tab. Adjust the field of view and matrix size to 105 $x 105$ to ensure a resolution of $333 \mu \mathrm{m} \times 333 \mu \mathrm{m}$.

3. Set the slice orientation to axial and the number of slices to 25 , resulting in a slice thickness of $500 \mu \mathrm{m}$ and interslice distance of 600 $\mu \mathrm{m}$. Amend the readout direction into left-right.

4. Click the Contrast tab to adjust the echo time to $24 \mathrm{~ms}$ and repetition time to $6,250 \mathrm{~ms}$.

5. Set the bandwidth to $250,000 \mathrm{~Hz}$ and turn the fat suppression on. Adjust the number of averages to one.

6. Click on the Research tab and change the number of averages (EPI segments) to 4 .

7. Click on the Diffusion tab within the research tab. Perform this step separately for each of the three diffusion shells.

1. Adjust the number of diffusion directions to 32 for the first shell, 46 for the second shell, and 64 for the third shell.

2. Adjust the gradient directions with custom gradient directions files.

3. Change the number of $B 0$ images to 5 for the first shell, 5 for the second shell, and 7 for the third shell.

4. Adjust the b-value per direction to $800 \mathrm{~s} / \mathrm{mm}^{2}$ for the first shell, $1500 \mathrm{~s} / \mathrm{mm}^{2}$ for the second shell, and $2000 \mathrm{~s} / \mathrm{mm}^{2}$ for the third shell.

NOTE: Adjusting the gradient directions with a custom gradient directions file can be done manually by setting Enter Diffusion Directions to yes or automatically by using the DTI_SET_DIRECTIONS macro.

8. Open the Geometry editor and place the field of view between the bulbus and cerebellum containing only the cerebrum to reduce artifact and scan time. Position six saturation bands of $5 \mathrm{~mm}$ outside the brain to reduce artifacts by clicking on Saturation and sliding the bands in the preferred position using the scroll bars.

NOTE: The bulbus and cerebellum can be identified based on anatomical landmarks and the three images of the tripilot scan.

9. Acquire the imported sequences by clicking on the traffic light symbol. Using the settings of the parameters described above, the acquisition time of the T2-RARE scan is $12 \mathrm{~min}$, of the first DWI shell $15 \mathrm{~min}$, of the second DWI shell $21 \mathrm{~min}$ and of the third shell $30 \mathrm{~min}$. The total acquisition time is approximately $80 \mathrm{~min}$ (on a single receiver channel system).

10. At the completion of the scanning protocol, remove the animal from the scanner bed, and place the animal in a clean cage with a heating pad at $37^{\circ} \mathrm{C}$. Return the animal to the home cage when it regains consciousness.

\section{Image processing}

NOTE: In the following sections, the processing of the diffusion images is described in MRtrix 3 , ExploreDTI ${ }^{19}$ and Amide software ${ }^{20}$ which are open access toolboxes. However, the preprocessing steps can be performed in other toolboxes (e.g., FSL, MedInria, DTIStudio).

1. Transfer the acquired data from the acquisition console by exporting the 2 dseq file.

2. Convert the 2 dseq files (raw DWI files) into the .mif format, which is the standard formatting of MRtrix 3 , to allow for further preprocessing steps in MRtrix3. Moreover, concatenate the three diffusion shells using the following commands in the shell: convert_bruker pdata/1/2dseq ratID_T2.mih (for the T2-weighted images) convert_bruker pdata/1/2dseq ratID_dwi1.mih (for the first diffusion shell) convert bruker pdata/1/2dseq ratID_dwi2.mih (for the second diffusion shell) convert_bruker pdata/1/2dseq ratID_dwi3.mih (for the third diffusion shell) mrcat ratID_dwi1.mif ratID_dwi2.mif ratID_dwi3.mif ratID_dwi.mif

3. Perform noise correction and Gibbs ringing correction on the DWIs in MRtrix ${ }^{21,22}$. Also, convert the corrected DWI images and T2 image to NIFTI format using the following commands:

dwidenoise ratID_dwi.mif ratID_dwi_denoised.mif

mrdegibbs ratID_dwi_denoised.mif ratID_dwi_denoised_gr.mif

mrconvert ratID dwi denoised gr.mif ratID.nii

mrconvert ratID_T2.mif ratID_T2.nii

4. Perform correction for EPI, motion and Eddy current distortions in ExploreDTI:

1. Convert the NIFTI images to a .mat file by clicking Calculate DTI*.mat file | Convert raw data to DTI*.mat file. Change the diffusion tensor estimation to weighed linear and the b-value to NaN. Adjust the voxel size to 0.3330 .3330 .6 , number of non-DWI images to 17 , number of DWI images to 142, and matrix size to 10510525. 
NOTE: By setting the b-value to NaN, ExploreDTI will regard the dataset as a kurtosis data set.

2. Click on the Settings tab to adjust the settings for EPI correction (this is turned off by default). Select SM/EC/EPI correction, also register to other data? and click on Yes, to do the EPI correction (non-rigid). Specify the suffix of the anatomical T2 image corresponding to the diffusion data set.

NOTE: ExploreDTI corrects for EPI distortions using image registration between the undistorted anatomical image and the diffusion image.

3. Click on the Plugins tab and select Correction for subject motion \& EC/EPI distortions and select the preprocessed diffusion data file from step 2.3. Make sure that the T2 image is in the same folder and has the same base as the diffusion data file name (e.g., rat1.nii for the DWI and rat1_T2.nii for the anatomical image). This step will generate a "native" ("native.mat) and "transformed" file (*trafo.mat).

5. Calculate the DTI metrics for each rat by clicking Plugins and Export stuff to *.nii and selecting the parametric maps of the DTI model: fractional anisotropy (FA), mean diffusivity (MD), radial diffusivity (RD), and axial diffusivity (AD; denoted as "largest eigenvalue L1").

6. Additionally, export the parametric maps for the kurtosis model (MK, AK, and RK) and WMTI model (AWF, AxEAD, RadEAD and TORT). Processing of the diffusion images will result in 12 parametric maps (Figure 1, Figure 2, Figure 3) that can be used for further microstructural analysis.

7. Create a mask file for the hippocampus of each rat using MRtrix3.

1. Load the FA image of the rat in the MRtrix viewer by clicking Tool and ROI editor.

2. Create a new ROI by clicking the "+" button and press Edit to draw the ROI on each slice that includes the hippocampus (Figure 4). To erase unwanted areas from the ROI drawn, press the right mouse button.

3. When the drawing of the ROI is completed, save the mask image by clicking the Save button.

NOTE: This mask file will be a binary NIFTI image file with voxels of value 1 containing the hippocampal tissue, and remaining voxels will have values of 0 . To standardize the region of the hippocampus across rats, the parametric maps can be co-registered with a study specific template with predefined regions of interest delineated ${ }^{23}$ or a rat brain atlas.

8. To extract the diffusion metrics of the hippocampus of the rat, use the created mask file of step 4.6 and open the Amide software.

1. Open the parametric maps and mask image of the rat.

2. To add the ROI of the mask file into Amide, select the mask file image, click Edit | Add ROI | 3D Isocontour and click on the ROI displayed in the mask image. Give the ROI a meaningful name and confirm that this volume should only contain voxels having a value of one.

3. To calculate the mean values of the diffusion metrics in the hippocampus, click Tools | Calculate RoI Statistics and indicate the images and ROI that should be included. After clicking Execute, another screen will pop up with computed values that can be used for further statistical analysis. This file can either be saved or copied into a preferred data format (e.g., .xlsx or .csv file).

\section{Statistical analysis}

NOTE: In the following sections, we describe processing of the diffusion images in SPSS Statistics 24; however, the statistical analysis can be performed in other statistical toolboxes.

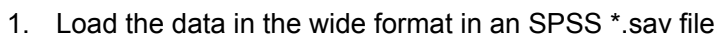

2. To test for statistical differences between the two groups for each timepoint (i.e., baseline or 1-day post-injury), click Analyze | Nonparametric tests | Legacy Dialogs | 2 Independent Samples Tests. Load the variables that need to be tested and specify the groups (i.e., TBI and sham groups). Indicate the Mann-Whitney $U$ as the test type.

3. To test for statistical differences between the 2 time point within each group the data file needs to be split. Go to Go to Data, Split File and indicate Compare groups. Next, click on Analyze, Nonparametric tests, Legacy Dialogs, 2 Related Samples Tests, load the variables that need to be compared and indicate Wilcoxon as test type.

NOTE: To correct for multiple comparisons, p-values are adjusted for each diffusion model using Bonferroni correction [i.e., p-value divided by the number of parameters compared (DTI 4, DKI 3, and WMTI 4)]. More specifically, $p<0.0125$ is considered significant for the DTI and WMTI models, and $p<0.016$ is considered significant for the DKI model.

\section{Representative Results}

In the study, all TBI rats $(n=10)$ survived the impact and were able to recover from the impact and anesthesia within 15 min after detachment from anesthesia ${ }^{23}$. On the CT images, there was no evidence of skull fractures and the T2 images did not show any abnormalities such as bleeding, enlarged ventricles, or edema formation at the contusion site 1 day after trauma (Figure 5). Thus, based on these visual inspections of the anatomical images, large focal lesions were not detected, confirming the diffuse and mild nature of the injury.

The quality of the coregistration (non-rigid) step between the T2 image and diffusion data set (step 4.4) was examined by adding an overlay of the T2 image to the color-encoded FA map (Figure 6). Then, the FA, MD, AD, and RD parametric maps were calculated (Figure 1) and loaded into the Amide software. Based on the FA map, a ROI including the hippocampal structure was drawn (Figure 4). Statistical values of the diffusion metrics were calculated averaged over all voxels within the region of interest and the mean values of each DTI metric were exported for further analysis. Another quality check of the diffusion data can be performed by inspecting the outliers in the DTI metrics. For example, FA values in the hippocampus should be around 0.15 ; therefore, values of $<0.10$ (denoting isotropic diffusion) or $>0.30$ (values are seen in white matter) can be regarded as biologically implausible values. These datapoints should be rejected from further analysis. Also, the mean values for AK, RK, and MK of the diffusion kurtosis model as well as the AWF, AxEAD, RadEAD, and TORT of the WMTI model were calculated (Figure 2 , Figure 3). 
In our study, analysis of the DTI metrics revealed significant increased FA values ( $p=0.007)$, and decreased diffusivity values (MD and RD) ( $p$ $=0.007$ and $p=0.007$, respectively) following impact in the mTBI group (Figure 7). These decreases in RD and MD were significantly different from the sham group ( $p=0.005$ and $p=0.004$, respectively). Diffusion kurtosis metrics showed a significant decrease in RK ( $p=0.005)$ following impact but no changes in AK or MK (Figure 8). Using the WMTI model, RadEAD $(p=0.007)$ and TORT $(p=0.007)$ displayed a significant decrease and increase, respectively, in the mTBI group 1 day after the impact (Figure 9C,D). The values in the sham group did not show any significant changes.

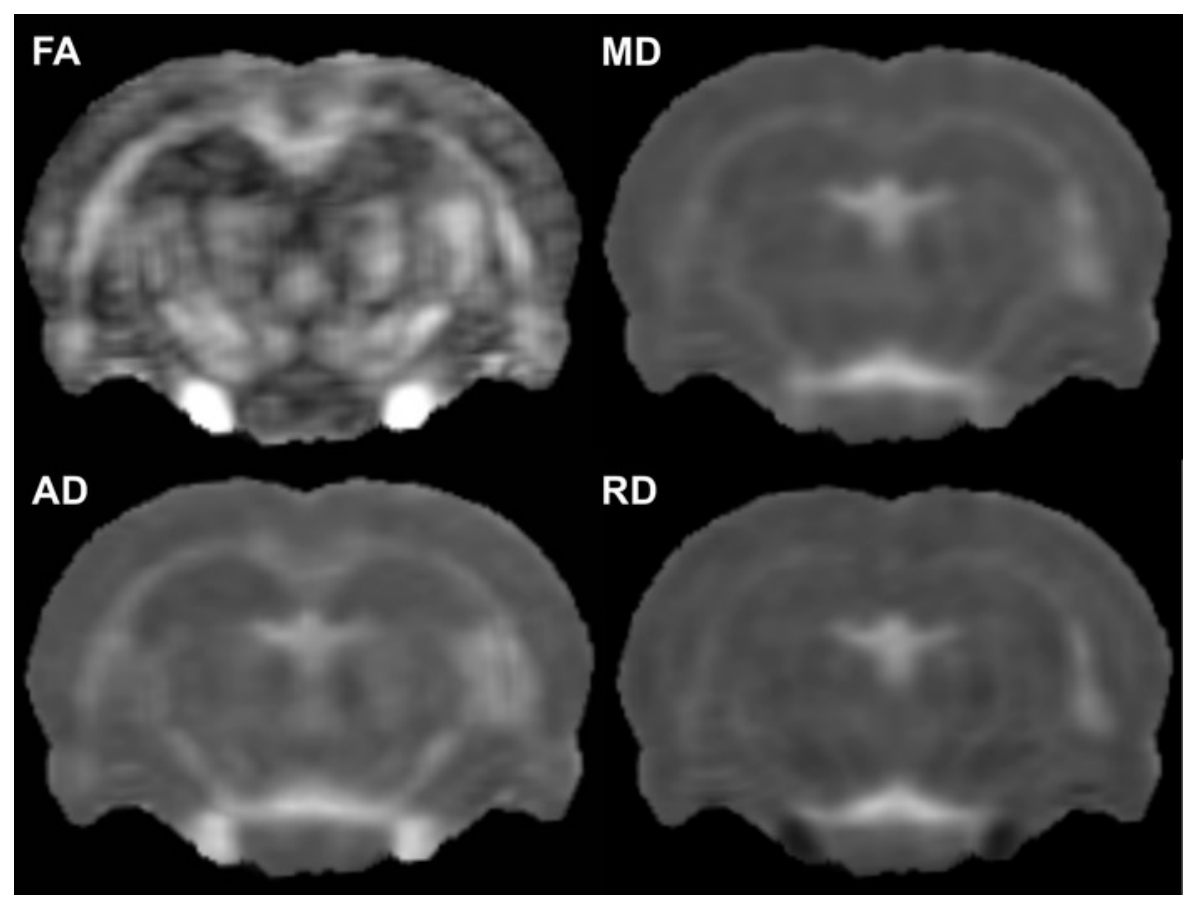

Figure 1: Representative parametric maps for fractional anisotropy (FA), mean diffusivity (MD), axial diffusivity (AD), and radial diffusivity (RD). Please click here to view a larger version of this figure.

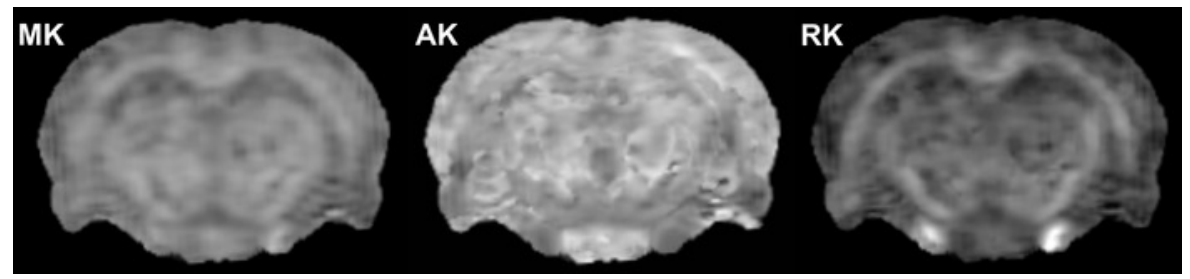

Figure 2: Representative parametric maps for mean kurtosis (MK), axial kurtosis (AK), and radial kurtosis (RK). Please click here to view a larger version of this figure. 


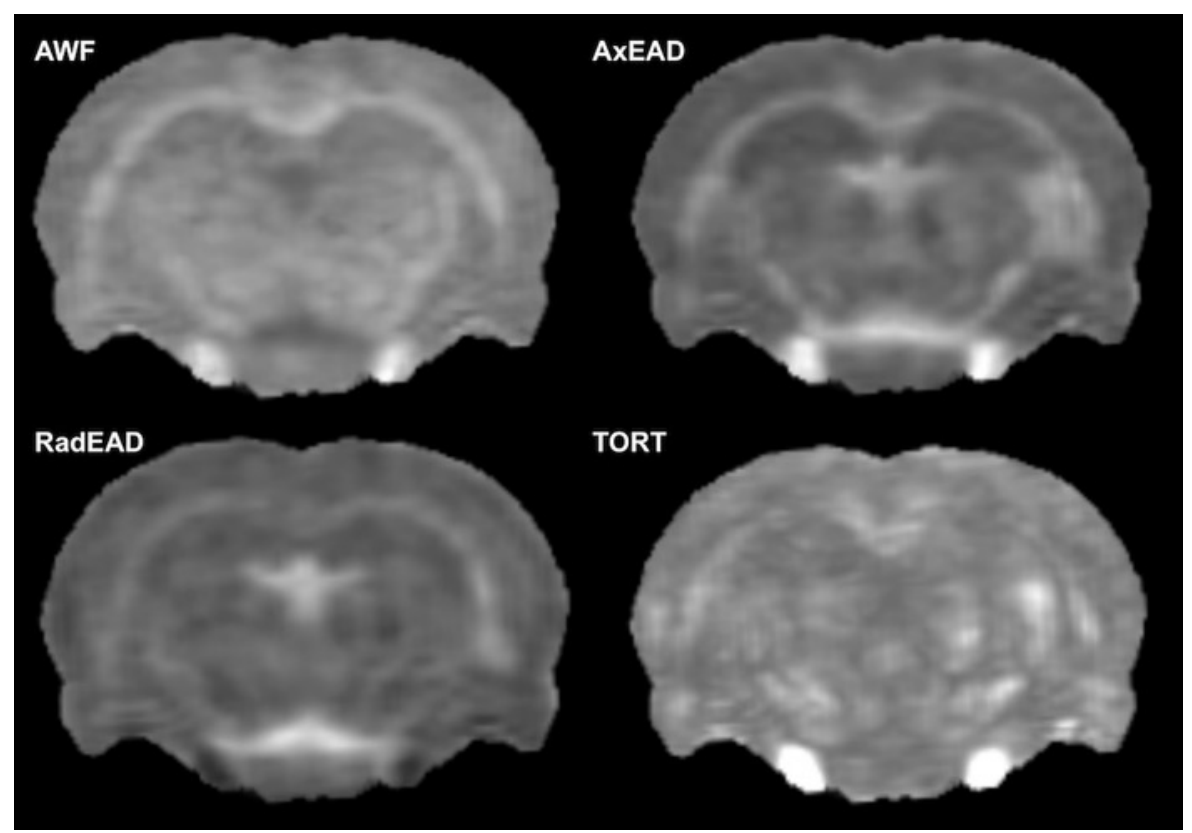

Figure 3: Representative parametric maps for axonal water fraction (AWF), axial and radial extra axonal diffusivity (AxEAD, RadEAD), and tortuosity (TORT). Please click here to view a larger version of this figure.

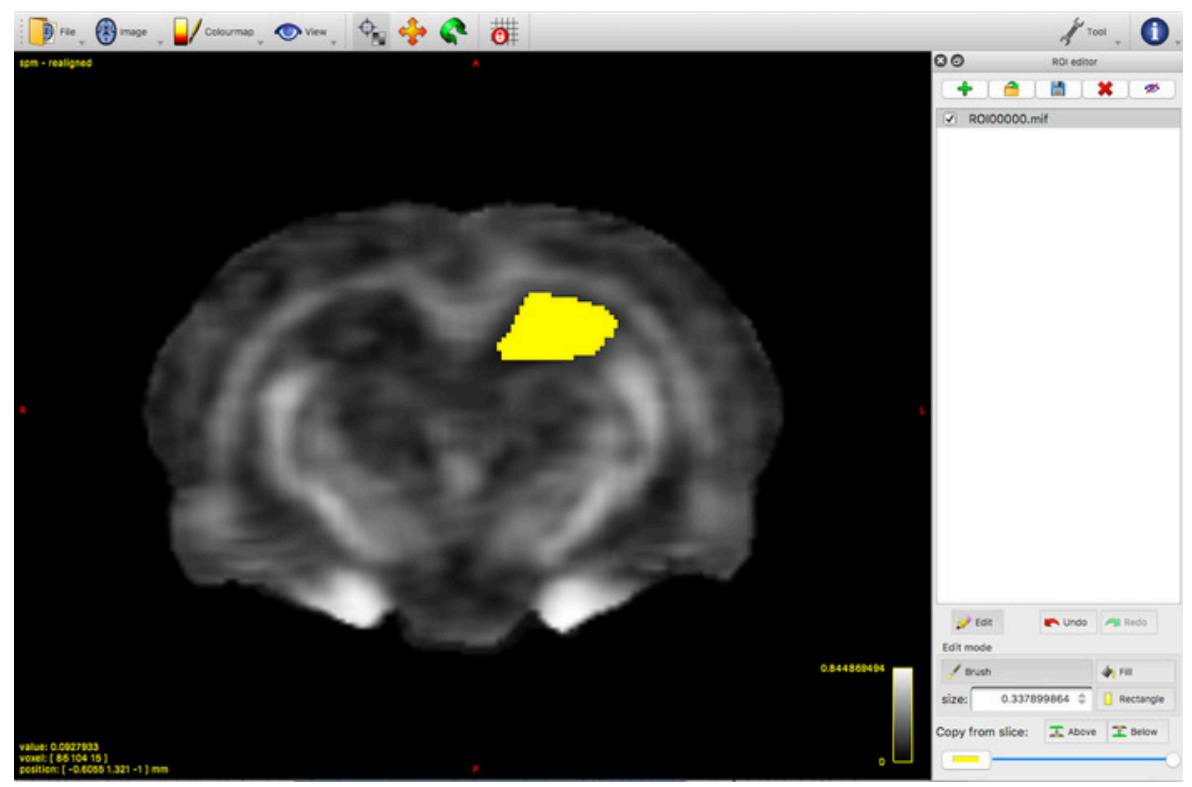

Figure 4: Creating a mask in MRtrix3. A ROI is drawn around the hippocampus on all slices containing the volume of the hippocampus, and the volume is saved as a mask file. This can either be done for each rat individually or by using a study specific template mask file to which each of the parametric maps can be co-registered. Please click here to view a larger version of this figure. 


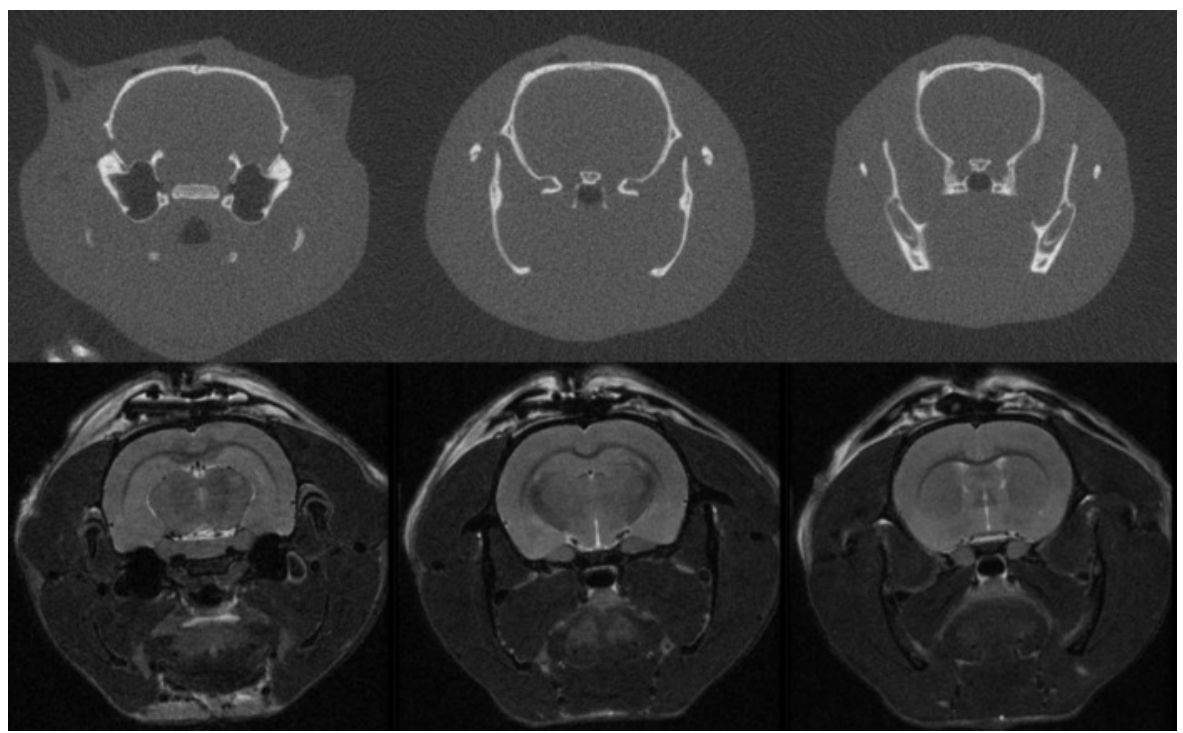

Figure 5: CT and T2 weighted images of a representative mTBI animal 1 day after impact. The CT images (top row) do not show any skull fractures. On the T2-weighted images (bottom row) no bleeding, enlarged ventricles, or edema formation were demonstrated. Of note, edema formation is clearly visible as a hyperintense area around the wound area from the surgical intervention. Please click here to view a larger version of this figure.

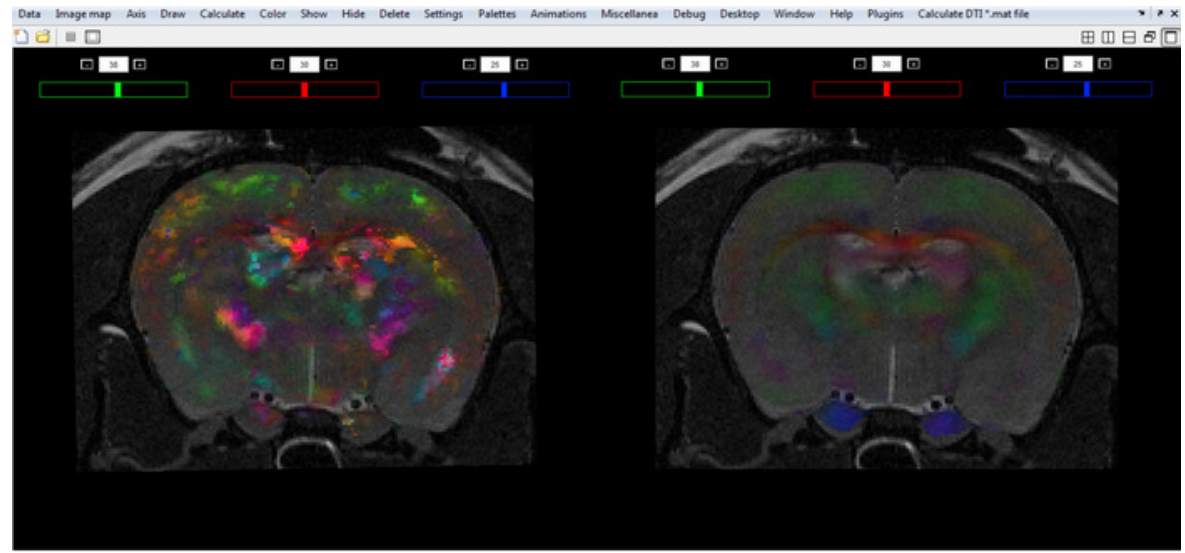

Figure 6: Color encoded FA map of diffusion data set overlaid with the anatomical image after correction for EPI, motion, and Eddy current correction in ExploreDTI. Shown is a bad correction and co-registration on the left and good examples on the right. It should be ensured that the color encoding is correct: left-right direction in red (e.g., corpus callosum), anterior-posterior direction in green, and inferiorsuperior direction in blue (e.g., cingulum). Additionally, the color encoded FA image should be perfectly aligned with the anatomical image. Please click here to view a larger version of this figure.
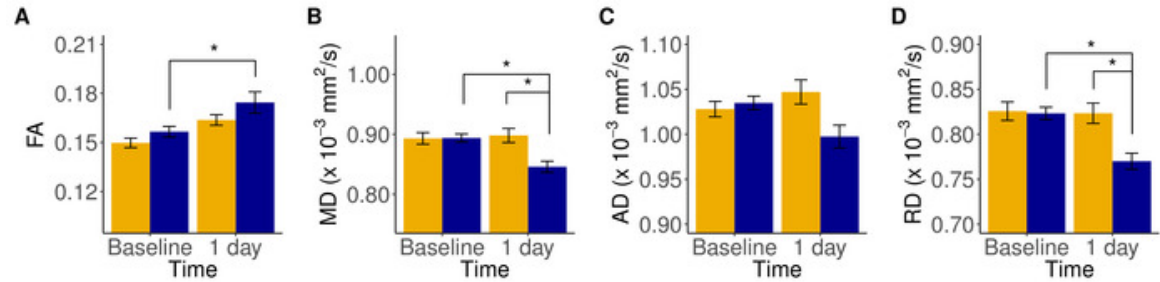

\section{Sham ImTBI}

Figure 7: Changes in diffusion tensor metrics of hippocampus for sham $(n=10)$ and $m T B I$ animals $(n=10)$. Following impact, there was a significant increase in FA (A) and significant decreases in mean diffusivity (B) and radial diffusivity (D) in the mTBI animals (B,D). No significant differences were observed for axial diffusivity $(\mathbf{C})$ in the $\mathrm{mTBI}$ rats. The sham animals did not show any significant DTI changes $\left({ }^{*} p<0.0125\right)$.

Please click here to view a larger version of this figure. 
A

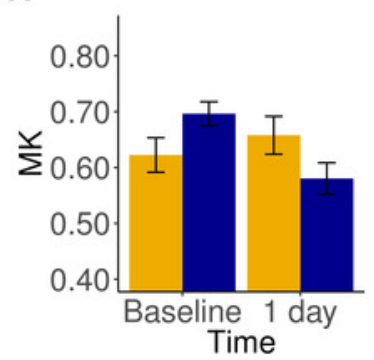

B

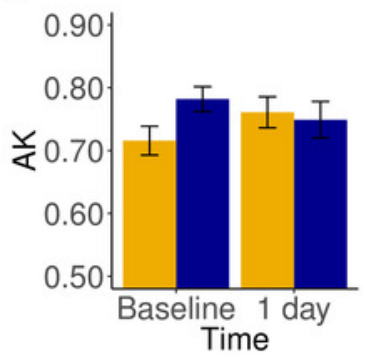

C

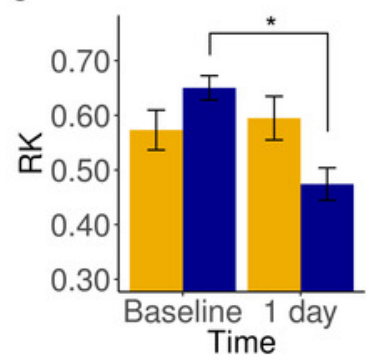

\section{Sham $1 \mathrm{mTBI}$}

Figure 8: Changes in diffusion kurtosis metrics of hippocampus for sham $(n=10)$ and $\mathrm{mTBI}$ animals $(\mathrm{n}=10)$. Following impact, there was a significant decrease in RK (C) of the mTBI animals but no changes in AK (B) or MK (A). The sham animals did not show any changes ( ${ }^{*} p$ $<0.0166)$. Please click here to view a larger version of this figure.
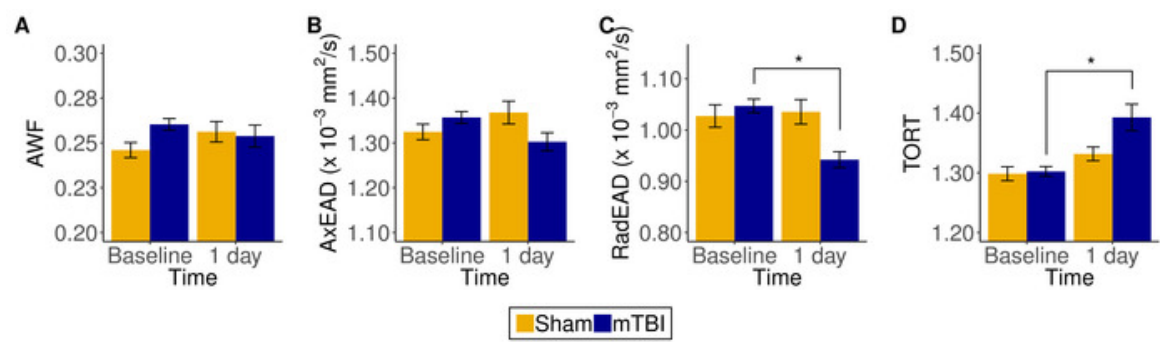

$$
\text { Sham ImTBI }
$$

Figure 9: Changes in white matter tract integrity metrics of hippocampus for sham $(n=10)$ and $m$ TBI animals $(n=10)$. Following impact, there was a significant decrease in RadEAD (C) and significant increase in TORT (D) of the mTBI animals but no change in AWF or AxEAD $(A, B)$. The sham animals did not show any changes $\left({ }^{*} \mathrm{p}<0.0125\right)$. Please click here to view a larger version of this figure.

\section{Discussion}

Since mTBI often is the result of a diffuse and subtle injury that shows no abnormalities on CT and conventional MRI scans, the evaluation of microstructural damage after a mild trauma remains a challenge. Therefore, more advanced imaging techniques are needed to visualize the full extent of the trauma. The application of diffusion magnetic resonance imaging in TBI research has gained more interest during the last decade, where diffusion tensor imaging is most frequently used ${ }^{5}$. A limitation of the DTI model is the assumption of a Gaussian diffusion process that is not a precise assumption for brain microstructure (consisting of a complex network of axons and cells with membranes acting as barriers), resulting in DTI metrics non-specific to the underlying biological microstructure ${ }^{24}$. Diffusion kurtosis imaging is an extension of the DTI model and attempts to characterize the degree of non-Gaussian diffusion ${ }^{17}$. This may provide additional information about tissue heterogeneity or complexity.

Though, a drawback of DTI and DKI models is that they are only a representation of the diffusion signal, which characterizes the probabilistic water displacement profile but is not specific to microstructure ${ }^{6}$. On the other hand, the white matter tract integrity model based on the kurtosis tensor is a microstructural mapping technique that incorporates a priori biological information (assumptions) into the model ${ }^{18}$. It attributes the diffusion signal to tissue compartments and can assess biological attributes more directly. These biophysical models may thus offer new information for describing abnormalities after $\mathrm{mTBI}$ and overcome this non-specificity issue ${ }^{6}$. Using these three different models, microstructural alterations and biological processes were able to be visualized following mTBI in more detail, specifically by using the Marmarou weight drop model.

The Marmarou weight drop model is easy to use and requires only minor surgery; however, a second experimenter is recommended to move the rat away from the glass tube immediately after the first impact to avoid a second one. Additionally, it is sometimes required to help the rat regain its breathing reflex following the impact. The rather long MRI protocol, with a total acquisition time of around 80 min, is well-tolerated by both the sham and mTBI rats. Though, during scanning, it is important to monitor breathing cycle and adjust the anesthesia if the animal is sleeping too deeply or lightly. It is also important to keep the animal warm both during and after the acquisition until the rat is fully awake to avoid hypothermia.

In advanced diffusion MRI, movement artifacts should be avoided as much as possible. A simple solution to reduce movement during scanning is to make use of a teeth bar and fixate the head with a small piece of tape or two ear bars, if available. This ensures that the head will not move up and down every time the rat takes a breath.

Using advanced diffusion MRI protocols, the acquired images must pass through several (pre-) processing steps, mostly using different software tools, before they can be used for further analysis. A drawback of using different software tools to process the diffusion-weighted images is that (often) each tool uses its own data format to encode the gradient directions table. MRtrix 3 stores the gradient information together with the diffusion weighted image in a .mif file, while ExploreDTI makes use of a separate file (B-matrix) to store the gradient directions. Therefore, it is important to check that the gradient directions are correctly transferred from MRtrix 3 to ExploreDTI. This can be done by checking that color encoding is correct on color encoded FA images [i.e., left-right direction in red (e.g., corpus callosum), anterior-posterior direction in green, 
and inferior-superior direction in blue (e.g., cingulum)]. The color encoded FA images can also be used to check the quality of the non-rigid coregistration process between the diffusion-weighted images and structural T2- weighted images.

Using ExploreDTI, parametric maps were extracted using the DTI, DKI, and WMTI models. The DTI model provided parametric maps for MD, $A D, R D$, and FA, while the DKI model provides parametric maps for MK, AK, and RK. Although four metrics of the WMTI model were calculated (i.e., AWF, AxEAD, RadEAD, TORT), it was not possible to extract intra-axonal diffusivity (IAD) within ExploreDTI. IAD can be obtained using a MATLAB tool provided by the developers of the WMTI model ${ }^{25}$. To do so, the diffusion-weighted images and gradient information must be transferred again from ExploreDTI into Matlab. This step is again prone to errors concerning the encoding of gradient information. Additionally, the kurtosis tensor and the WMTI parameters must be estimated and calculated again.

Preprocessing of the acquired images, estimation of the tensors, and calculation of the parametric maps requires a long period of computing time. Corrections for EPI, motion, and eddy current required $\sim 40$ min per data set on a server with eight cores and 16 GB RAM. Using a ROI analysis, mean values within the hippocampus were calculated before and 1 day after impact. Changes in the DTI, DKI and WMTI metrics were then quantified in the mTBI group. However, in the DKI metrics and AWF of the WMTI model, large inter-subject variability was observed, which resulted in an unexpected difference in baseline values between the sham and mTBI groups. This is most likely the result of voxels containing biologically implausible values (outliers) within the investigated region and may be filtered out in future studies before calculation of the mean values in Amide.

In conclusion, this protocol demonstrates the feasibility of advanced diffusion MRI for investigating and quantifying microstructural alterations in the hippocampus in a rat model of mTBI. Using three different diffusion models, complementary information can be obtained about the underlying biological processes that contribute to the conditions after mTBI. This represents a step forward in the development of biomarkers for mTBI that may be sensitive enough to identify specific microstructural changes in the early phase after mild impact.

\section{Disclosures}

The authors have no conflicts of interest to disclose.

\section{Acknowledgments}

The authors would like to thank Research Foundation - Flanders (FWO) for supporting this work (Grant number: G027815N).

\section{References}

1. Carroll, L. J. et al. Systematic Review of the Prognosis After Mild Traumatic Brain Injury in Adults: Cognitive, Psychiatric, and Mortality Outcomes: Results of the International Collaboration on Mild Traumatic Brain Injury Prognosis. Archives of Physical Medicine and Rehabilitation. 95 (3), S152-S173 (2014).

2. Buck, P. W. Mild Traumatic Brain Injury: A Silent Epidemic in Our Practices. Health \& Social Work. 36 (4), 299-302 (2011).

3. Bodanapally, U. K., Sours, C., Zhuo, J., Shanmuganathan, K. Imaging of Traumatic Brain Injury. Radiologic Clinics of North America. 53 (4), 695-715 (2015).

4. Basser, P. J., Mattiello, J., LeBihan, D. MR diffusion tensor spectroscopy and imaging. Biophysical Journal. 66 (1), $259-267$ (1994).

5. Hulkower, M. B., Poliak, D. B., Rosenbaum, S. B., Zimmerman, M. E., Lipton, M. L. A Decade of DTI in Traumatic Brain Injury: 10 Years and 100 Articles Later. American Journal of Neuroradiology. 34 (11), 2064-2074 (2013).

6. Hutchinson, E. B., Schwerin, S. C., Avram, A. V, Juliano, S. L., Pierpaoli, C. Diffusion MRI and the detection of alterations following traumatic brain injury. Journal of Neuroscience Research. 96 (4), 612-625 (2018).

7. Wallace, E. J., Mathias, J. L., Ward, L. Diffusion tensor imaging changes following mild, moderate and severe adult traumatic brain injury: a meta-analysis. Brain Imaging and Behavior. 1-15 (2018).

8. Rutgers, D. R. et al. White Matter Abnormalities in Mild Traumatic Brain Injury: A Diffusion Tensor Imaging Study. American Journal of Neuroradiology. 29 (3), 514-519 (2008)

9. Bondi, C. O. et al. Found in translation: Understanding the biology and behavior of experimental traumatic brain injury. Neuroscience \& Biobehavioral Reviews. 58, 123-146 (2015).

10. Shultz, S. R. et al. The potential for animal models to provide insight into mild traumatic brain injury: Translational challenges and strategies. Neuroscience \& Biobehavioral Reviews. 76, 396-414 (2017)

11. Osier, N. D., Dixon, C. E. The Controlled Cortical Impact Model: Applications, Considerations for Researchers, and Future Directions. Frontiers in Neurology. 7 (AUG) (2016).

12. Lyeth, B. G. Historical Review of the Fluid-Percussion TBI Model. Frontiers in Neurology. 7 (DEC), 1-7 (2016).

13. Marmarou, A., Foda, M. A. A. -E., Brink, W. van den, Campbell, J., Kita, H., Demetriadou, K. A new model of diffuse brain injury in rats. Journal of Neurosurgery. 80 (2), 291-300 (1994).

14. Heim, L. R. et al. The Invisibility of Mild Traumatic Brain Injury: Impaired Cognitive Performance as a Silent Symptom. Journal of Neurotrauma. 34 (17), 2518-2528 (2017).

15. Zohar, O., Rubovitch, V., Milman, A., Schreiber, S., Pick, C. G. Behavioral consequences of minimal traumatic brain injury in mice. Acta Neurobiol Exp (Wars). 71 (1), 36-45 (2011).

16. Pierpaoli, C., Basser, P. J. Toward a quantitative assessment of diffusion anisotropy. Magnetic resonance in medicine : official journal of the Society of Magnetic Resonance in Medicine / Society of Magnetic Resonance in Medicine. 36 (6), 893-906 (1996).

17. Jensen, J. H., Helpern, J. A. MRI quantification of non-Gaussian water diffusion by kurtosis analysis. NMR in Biomedicine. 23 (7), 698-710 (2010).

18. Fieremans, E., Jens H., Jensen, J. A. H. White matter characterization with diffusional kurtosis imaging. Neurolmage. 58, 177-188 (2011).

19. Leemans, A. Explore DTI. (2019). 
20. Loening, A. M., Gambhir, S. S. AMIDE: A Free Software Tool for Multimodality Medical Image Analysis. Molecular Imaging. 2 (3), 131-137 (2003).

21. Veraart, J. et al. Denoising of diffusion MRI using random matrix theory. Neurolmage. 142, 394-406 (2016).

22. Veraart, J., Fieremans, E., Novikov, D. S. Diffusion MRI noise mapping using random matrix theory. Magnetic Resonance in Medicine. 76 (5), 1582-1593 (2016).

23. Braeckman, K. et al. Dynamic changes in hippocampal diffusion and kurtosis metrics following experimental mTBI correlate with glial reactivity. Neurolmage: Clinical. 21 (August 2018), 101669 (2019).

24. Jones, D. K., Knösche, T. R., Turner, R. White matter integrity, fiber count, and other fallacies: The do's and don'ts of diffusion MRI. Neurolmage. 73, 239-254 (2013).

25. Matlab code DKI and WMTI model. at <https://github.com/NYU-DiffusionMRI/Diffusion-Kurtosis-Imaging> (2019). 